\title{
Stability Analysis of Linear Fractional-Order Neutral Systems with Time Delay
}

\author{
Hong Li, Shou-ming Zhong, Hou-biao Li* \\ School of Mathematics Sciences, University of Electronic Science and Technology of China, Chengdu, PR China \\ Email address: \\ sichuanhong@163.com (Hong Li), zhongsm@uestc.edu.cn (Shou-ming Zhong), lihoubiao0189@163.com (Hou-biao Li) \\ ${ }^{*}$ Corresponding author
}

To cite this article:

Hong Li, Shou-ming Zhong, Hou-biao Li. Stability Analysis of Linear Fractional-Order Neutral Systems with Time Delay. Science Journal of Circuits, Systems and Signal Processing. Vol. 6, No. 1, 2017, pp. 1-5. doi: 10.11648/j.cssp.20170601.11

Received: October 30, 2016; Accepted: February 9, 2017; Published: March 4, 2017

\begin{abstract}
In this paper, we mainly study the Lyapunov asymptotical stability of linear and interval linear fractional order neutral systems with time delay. By applying the characteristic equations of these two systems, some simple sufficient Lyapunov asymptotical stability conditions are deserved, which are quite different from other ones in literature. In addition, some numerical examples are provided to demonstrate the effectiveness of our results.
\end{abstract}

Keywords: Interval Fractional-Order Neutral Systems, Time Delay, Characteristic Equation

\section{Introduction}

Fractional order systems have many obvious advantages since fractional order differential is more adequate to describe real word problems because it has more degrees of freedom. At the same time, a memory is also included in the model. Therefore, fractional order systems have gained important applications in various sciences such as signal processing, viscoelasticity, electroanalytical chemistry, electric conductance of biological systems, modeling of neurons, diffusion processes, damping laws, rheology physics, electrode electrolyte polarization, electromagnetic wave, etc. For more details, please see [1-4].

Time delay may have considerable impacts on the stability of the system because it often presents in real processes due to transportation of materials or energy. Thus, most fractional systems may contain delay terms, such as fractional order neutral systems or some other fractional order delay systems. If the system contains delays both in its states and in the derivatives of its states, then the system is called a neutral type delay system. Neutral type delay systems are very common in realities.

Stability analysis is one of the most important issues in the theory of differential equations and their applications for both deterministic and stochastic cases. Stability analysis of fractional differential equations is more complex than that of classical differential equations, because fractional derivatives are nonlocal and have weakly singular kernels. The stability analysis of time delay systems can be generally classified as two types: the time delay dependent criteria and the time delay independent stability. As there is no the upper limit to time delay, time delay independent results can be regarded as conservative in practice. Because of the complex definition of fractional order integral, the analysis of fractional order equations is more difficult than that of integral equations. Nowadays, various stability analysis techniques have been used to derive stability criteria for the fractional system. The most well-known one is Matignon's stability theorem [5]. This theorem permits us to determine the stability of the linear fractional order system through the location in the complex plane of the dynamic matrix eigenvalues of the state space like system representation. Matignon's theorem is the starting point of several results in the field of linear fractional order system stability analysis. In addition, Lambert functions approach ([6, 7]), Lyapunov's second approach [8], Matrix measure approach $([9,10])$, Bellman-Gronwall's approach ([11]) and LMI approach ([12]) are also used to investigate the stability of fractional order linear systems. All of these approaches have their own advantages and disadvantages. Recently, a finite-time stability analysis of fractional order 
time delay systems is firstly presented and reported on paper [13]. But till now, only a few papers studied the stability of fractional neutral systems with delay. Lyapunov approach of nonlinear fractional order neutral system were extended in paper [14]. However, it is difficult to use Lyapunov method to study the stability of fractional order neutral systems with delay for the complicated of the fractional derivatives. All of those have motivated our research.

In this paper, we are interested in the Lyapunov asymptotical stability of linear fractional order neutral systems with time delay. By using the characteristic equation of the system, some simple sufficient Lyapunov asymptotical stability conditions are deserved. In addition, we studied the Lyapunov asymptotical stability of interval linear fractional order neutral system with time delay. Finally, two examples are provided to demonstrate the effectiveness of our results.

The rest of the paper is organized as follows. In Section 2, we give some notations and lemma, recall some concepts and preparation results. In Section 3, using the characteristic equations of the systems, we study the Lyapunov asymptotical stability of linear and interval linear fractional order neutral systems with time delay. Some sufficient conditions are deserved. In Section 4, two numerical examples are provided.

\section{Problem Formulation and Preliminaries}

In this section, we introduce some notations, definitions, and preliminary facts needed in this paper.

The idea of fractional calculus has been known since the development of the regular calculus, with the first reference probably being associated with Leibniz and L'Hospital in 1695 where half-order derivative was mentioned. The differ-integral operator, denoted by ${ }_{a} D_{t}^{\alpha}$, is a combined differentiation and integration operator commonly used in fractional calculus, which is defined by

$$
{ }_{a} D_{t}^{\alpha}= \begin{cases}\frac{d^{\alpha}}{d t^{\alpha}}, & \alpha>0 \\ 1, & \alpha=0 \\ \int_{a}^{t}(d \tau)^{-\alpha}, & \alpha<0 .\end{cases}
$$

Beyond all doubt, there are different definitions for fractional derivatives, see [15]. The most commonly used definitions are the Grunwald-Letnikov, Riemann-Liouville and Caputo definitions. Riemann-Liouville and Caputo definitions are often used in pure mathematicians, and the last one is often adopted by applied scientists, because Caputo definitions is more convenient in engineering applications. The Caputo definition is sometimes called smooth fractional derivative in literature because it is suitable to be treated by the Laplace transform technique, while the Riemann-Liouville definition is unsuitable. Here we only discuss Caputo derivative, so in the rest of the paper,
$D^{\alpha}$ is used to denote the Caputo fractional derivative of order $\alpha$. Define

$$
D^{\alpha} f(t)=\frac{d^{\alpha} f(t)}{d t^{\alpha}}=\frac{1}{\Gamma(\alpha-m)} \int_{0}^{t} \frac{f^{(m)}(\tau)}{(t-\tau)^{\alpha+1-m}} d \tau,
$$

where $m$ is an integer satisfying $m-1<\alpha \leq m$. In engineering physics and economics, the fractional order $\alpha$ often lies in $(0,2)$, so in this paper we always suppose on the case that the fractional order is $0<\alpha<2$.

Firstly, let us consider the linear fractional order neutral system with time delay described by the following form:

$$
\frac{d^{\alpha}}{d t^{\alpha}}(E x(t)-C x(t-\tau))=A x(t)+B x(t-\tau)
$$

with the initial condition $x\left(t_{0}+t\right)=\psi(t) \in C[-\Delta, 0]$. Here $0<\alpha<1$ is the fractional commensurate order, $x(t) \in \mathbb{R}^{n}$ denotes the state vector. $E, A$ and $B \in \mathbb{R}^{n \times n}$ are the constant matrices, and matrix $E$ is singular, that means rank $E=n_{1}<n$, and $\tau>0$ is the pure time delay.

If matrices $A$ and $B$ are uncertain, then the interval linear fractional order system with time delay above can be described by the state space equation of the following form

$$
\frac{d^{\alpha}}{d t^{\alpha}}(E x(t)-C x(t-\tau))=A x(t)-B x(t-\tau)
$$

where

$$
\begin{aligned}
& A \in\left[A^{1}, A^{2}\right]=\left\{\left[a_{i j}\right]: a_{i j}^{1} \leq a_{i j} \leq a_{i j}^{2},\right. \\
& B \in\left[B^{1}, B^{2}\right]=\left\{\left[b_{i j}\right]: b_{i j}^{1} \leq b_{i j} \leq b_{i j}^{2}, 1 \leq i, j \leq n\right\} .
\end{aligned}
$$

This kind of matrices are called interval matrices.

Throughout this article, let $\rho(A)$ be the spectral radium of the matrix $A,|\mathrm{~A}|$ denote the modulus matrix of the matrix $A$, and let $\mathfrak{R} e(s)$ be the real part of $s$.

First, let us recall a known lemma about matrix theory. To prove the main results in the next section, we need this very important lemma.

Lemma 2.1 ([15]). Let $R, T$, and $V \in \mathbb{C}^{n \times n}$. If $|R| \leq V$, then

$$
\begin{gathered}
\rho(R+T) \leq \rho(|R+T|) \leq \rho(|R|+|T|) \leq \rho(V+|T|), \\
\rho(R T) \leq \rho(|R \| T|) \leq \rho(V|T|), \\
\rho(R) \leq \rho(|R|) \leq \rho(V) .
\end{gathered}
$$

\section{Main Results}

In this section, we consider the stability of linear fractional neutral system (1) and interval linear fractional neutral system (2). Here, we always assume that these two fractional neutral systems have unique continuous solutions 


\subsection{Stability of Linear Fractional Neutral Systems with Time Delay}

In this subsection, several sufficient conditions of stability of linear fractional order neutral systems with time delay are given.

Theorem 3.1. If all the roots of the following characteristic equation

$$
D(s)=\operatorname{det}\left(s^{\alpha}\left(E-C e^{-s \tau}\right)-\left(A+B e^{-s \tau}\right)\right)=0
$$

have negative real parts, then the zero solution of the system (1) is Lyapunov asymptotically stable.

Proof. Similar to [16], by taking Laplace transform of the linear fractional order system (1), we can easily prove this theorem.

Next, we assume the matrix pair $(E, C)$ is regular with index one, then there exist nonsingular matrices $P, Q \in \mathbb{R}^{n \times n}$ such that

$$
P E Q=\left(\begin{array}{cc}
I_{n_{1}} & 0 \\
0 & 0
\end{array}\right), P C Q=\left(\begin{array}{cc}
C_{1} & 0 \\
0 & I_{n-n_{1}}
\end{array}\right)
$$

In addition, let $H(s)=\left(s^{\alpha}\left(E-C e^{-s \tau}\right)-A\right)^{-1}$, and $H_{M}$ is the matrix formed by taking the maximum magnitude of each element of matrix $H(s)$ for $\mathfrak{R} e(s)>0$. Then we have the following theorem.

Theorem 3.2. The system (1) is Lyapunov asymptotically stable, if the following inequalities are satisfied:

$$
\begin{gathered}
\text { (1). } \operatorname{det}\left(s^{\alpha}\left(E-C e^{-s \tau}\right)-A\right) \neq 0, \forall \tau>0, \Re e(s) \geq 0 ; \\
\text { (2). } \rho\left(H_{M}(|B|)<1 .\right.
\end{gathered}
$$

Proof. If the condition (1) in Theorem 3.2 holds, for $\mathfrak{R} e(s) \geq 0$, we have

$$
\begin{aligned}
D(s) & =\operatorname{det}\left(\left(s^{\alpha}\left(E-C e^{-s \tau}\right)-A\right)-B e^{-s \tau}\right) \\
& =\operatorname{det}\left(s^{\alpha} \tilde{E}-A\right) \operatorname{det}\left(I-\left(s^{\alpha} \tilde{E}-A\right)^{-1}\left(B e^{-s \tau}\right)\right) .
\end{aligned}
$$

Here $\tilde{E}=E-C e^{-s \tau}$.

If the condition (2) in Theorem 3.2 holds, using the Lemma 2.1 , we can obtain, for any $\mathfrak{R} e(s) \geq 0$

$$
\begin{aligned}
& \rho\left(\left(s^{\alpha}\left(E-C e^{-s \tau}\right)-A\right)^{-1} B e^{-s \tau}\right) \leq \rho\left(\left|F(s) \| B e^{-s \tau}\right|\right) \\
& \leq \rho\left(H_{M}|B|\right)<1 .
\end{aligned}
$$

According to (4) and (5), we can get

$$
\operatorname{det}\left(I-\left(s^{\alpha}\left(E-C e^{-s \tau}\right)-A\right)^{-1}\left(B e^{-s \tau}\right)\right) \neq 0, \forall \Re \mathrm{e}(\mathrm{s}) \geq 0 .
$$

So $D(s) \neq 0$ for $\Re e(s) \geq 0$ and $s \neq 0$. Thus, when that two conditions in Theorem 3.2 hold, we can obtain that all the roots of the following characteristic equation

$$
D(s)=\operatorname{det}\left(s^{\alpha}\left(E-C e^{-s \tau}\right)-\left(A+B e^{-s \tau}\right)\right)=0
$$

have negative real parts, so the linear fractional order neutral system (1) is Lyapunov asymptotically stable. Thus the proof is completed.

Theorem 3.3. The system (1) is Lyapunov asymptotically stable, if for any $\tau>0, \forall \Re \mathrm{e}(\mathrm{s}) \geq 0$ the following inequalities are satisfied

$$
\begin{aligned}
& \text { (1). } \operatorname{det}\left(s^{\alpha}\left(\begin{array}{cc}
I_{n_{1}}-C_{1} e^{-s \tau} & 0 \\
0 & -e^{-s \tau} I_{n-n_{1}}
\end{array}\right)-P A Q\right) \neq 0 \text {; } \\
& \text { (2). } \rho\left(G_{M}(|B|)<1,\right.
\end{aligned}
$$

where $G_{M}$ is the matrix formed by taking the maximum magnitude of each element of the following matrix

$$
G_{M}(s)=\left(s^{\alpha}\left(\begin{array}{cc}
I_{n_{1}}-C_{1} e^{-s \tau} & 0 \\
0 & -e^{-s \tau} I_{n-n_{1}}
\end{array}\right)-P A Q\right)^{-1}
$$

Proof. If the condition (1) in Theorem 3.3 holds, then the following matrix

$$
\left(s^{\alpha}\left(\begin{array}{cc}
I_{n_{1}}-C_{1} e^{-s \tau} & 0 \\
0 & -e^{-s \tau} I_{n-n_{1}}
\end{array}\right)-P A Q\right)^{-1}
$$

exists for $\mathfrak{R} e(s) \geq 0$. According to (3), we have

$$
\begin{gathered}
D(s)=\operatorname{det}\left(\left(s^{\alpha}\left(E-C e^{-s \tau}\right)-A\right)-B e^{-s \tau}\right) \\
=\operatorname{det}\left(P^{-1}\right) \operatorname{det}\left(Q^{-1}\right) \operatorname{det}\left(\left(s^{\alpha} \tilde{P}-P A Q\right)-P B Q e^{-s \tau}\right) \\
=\operatorname{det}\left(P^{-1}\right) \operatorname{det}\left(Q^{-1}\right) \operatorname{det}\left(s^{\alpha}\left(\begin{array}{cc}
I_{n_{1}}-C_{1} e^{-s \tau} & 0 \\
0 & -e^{-s \tau} I_{n-n_{1}}
\end{array}\right)-P A Q\right) \\
\operatorname{det}\left(I-\left(s^{\alpha}\left(\begin{array}{cc}
I_{n_{1}}-C_{1} e^{-s \tau} & 0 \\
0 & e^{-s \tau} I_{n-n_{1}}
\end{array}\right)-P A Q\right)^{-1} P B Q e^{-s \tau}\right)
\end{gathered}
$$

Here $\tilde{P}=P E Q-P C Q e^{-s \tau}$.

If the condition (2) in Theorem 3.3 holds, then using the Lemma 2.1, we can obtain

$$
\begin{aligned}
& \rho\left(\left(s^{\alpha}\left(\begin{array}{cc}
I_{n_{1}}-C_{1} e^{-s \tau} & 0 \\
0 & e^{-s \tau} I_{n-n_{1}}
\end{array}\right)-P A Q\right)^{-1} P B Q e^{-s \tau}\right) \\
& \leq \rho\left(G_{M}(|P B Q|)\right)<1 .
\end{aligned}
$$

Then, according to (6) and (7), we can get

$$
\operatorname{det}\left(I-\left(s^{\alpha}\left(\begin{array}{cc}
I_{n_{1}}-C_{1} e^{-s \tau} & 0 \\
0 & e^{-s \tau} I_{n-n_{1}}
\end{array}\right)-P A Q\right)^{-1} P B Q e^{-s \tau}\right) \neq 0 .
$$


So we have $D(s) \neq 0$ for any $\mathfrak{R} e(s) \geq 0$. Thus we complete the proof.

\subsection{Stability of Interval Linear Fractional Order Neutral System with Time Delay}

In this subsection, several sufficient conditions of stability of interval linear fractional order neutral systems are given.

Now, we consider the interval linear fractional order neutral system (2). Start with, we need to give some definitions.

Let

$$
\begin{aligned}
& \bar{A}=\frac{1}{2}\left(A^{1}+A^{2}\right), \Delta A=A-\bar{A}, A_{M}=A_{2}-\bar{A}, \\
& \bar{B}=\frac{1}{2}\left(B^{1}+B^{2}\right), \Delta B=B-\bar{B}, B_{M}=B_{2}-\bar{B},
\end{aligned}
$$

then we can get $|\Delta B| \leq B_{M}$. We have the following theorems about the interval linear fractional order neutral system (2).

Theorem 3.4. The interval linear fractional order neutral system (2) is Lyapunov asymptotically stable, if the following inequalities are satisfied:

$$
\begin{aligned}
& \text { (1). } \operatorname{det}\left(s^{\alpha}\left(E-C e^{-s \tau}\right)-\bar{A}\right) \neq 0, \tau>0, \Re e(s) \geq 0 ; \\
& \text { (2). } \rho\left(F_{M}\left(A_{M}+B_{M}+|\bar{B}|\right)<1 .\right.
\end{aligned}
$$

Proof. If the condition (1) in Theorem 3.4 holds, we have for $\mathfrak{R e}(s) \geq 0$,

$$
\begin{aligned}
D(s) & =\operatorname{det}\left(\left(s^{\alpha} \tilde{E}-\bar{A}\right)-\left(\Delta A+(\bar{B}+\Delta B) e^{-s \tau}\right)\right) \\
& =\operatorname{det}\left(s^{\alpha} \tilde{E}-\bar{A}\right) \operatorname{det}\left(I-\left(s^{\alpha} \tilde{E}-\bar{A}\right)^{-1}\left(\Delta A+(\bar{B}+\Delta B) e^{-s \tau}\right)\right) .
\end{aligned}
$$

If the condition (2) in Theorem 3.4 holds, using the Lemma 2.1 , we obtain, for any $\Re e(s) \geq 0$

$$
\begin{aligned}
& \rho\left(\left(s^{\alpha}\left(E-C e^{-s \tau}\right)-\bar{A}\right)^{-1}\left(\Delta A+(\bar{B}+\Delta B) e^{-s \tau}\right)\right) \\
& \leq \rho\left(\left|F(s) \| \Delta A+(\bar{B}+\Delta B) e^{-s \tau}\right|\right) \\
& \leq \rho\left(F_{M}\left(A_{M}+|\bar{B}|+B_{M}\right)\right)<1 .
\end{aligned}
$$

According to (8) and (9), we can get $D(s) \neq 0$ for $\Re e(s) \geq 0$, so when that two conditions in Theorem 3.4 hold, we can easily obtain that all the roots of the characteristic equation of system (2) have negative real parts, then the system (1) is Lyapunov asymptotically stable. Thus the proof is completed.

Theorem 3.5. The system (2) is Lyapunov asymptotically stable, if for any $\tau>0, \Re e(s) \geq 0$, the following two inequalities are satisfied

$$
\begin{aligned}
& \text { (1). } \operatorname{det}\left(\left(s^{\alpha}\left(\begin{array}{cc}
I_{n_{1}}-C_{1} e^{-s \tau} & 0 \\
0 & -e^{-s \tau}
\end{array}\right)-P \bar{A} Q\right)\right) \neq 0, \\
& \text { (2). } \rho\left(G_{M}\left(\left|P A_{M} Q\right|+\left|P\left(B_{M}+\bar{B}\right) Q\right|\right)<1,\right.
\end{aligned}
$$

where $G_{M}$ is the matrix defined in Theorem 3.3.

Proof. If the condition (1) in Theorem 3.5 holds, according to (3), we have

$$
\begin{aligned}
& D(s)=\operatorname{det}\left(s^{\alpha}\left(E-C e^{-s \tau}\right)-A-B e^{-s \tau}\right) \\
& =\operatorname{det}\left(\left(s^{\alpha}\left(E-C e^{-s \tau}\right)-\bar{A}\right)+\Delta A+(\bar{B}+\Delta B) e^{-s \tau}\right) \\
& =\operatorname{det}\left(P^{-1}\right) \operatorname{det}\left(Q^{-1}\right) \operatorname{det}\left(\left(s^{\alpha} \tilde{P}-P \bar{A} Q\right)+P\left(\Delta A+(\bar{B}+\Delta B) Q e^{-s \tau}\right)\right) \\
& =\operatorname{det}\left(P^{-1}\right) \operatorname{det}\left(Q^{-1}\right) \operatorname{det}\left(s^{\alpha}\left(\begin{array}{cc}
I_{n_{1}}-C_{1} e^{-s \tau} & 0 \\
0 & -e^{-s \tau} I_{n-n_{1}}
\end{array}\right)-P \bar{A} Q\right) \\
& \operatorname{det}\left(\left(I-s^{\alpha}\left(\begin{array}{cc}
I_{n_{1}}-C_{1} e^{-s \tau} & 0 \\
0 & e^{-s \tau} I_{n-n_{1}}
\end{array}\right)-P \bar{A} Q\right)^{-1}\left(P \Delta A Q+P(\bar{B}+\Delta B) Q e^{-s \tau}\right)\right) .
\end{aligned}
$$

If the condition (2) in Theorem 3.5 holds, using the Lemma 2.1, we obtain, for $\mathfrak{R} e(s) \geq 0$,

$$
\begin{aligned}
& \rho\left(\left(s^{\alpha}\left(\begin{array}{cc}
I_{n_{1}}-C_{1} e^{-s \tau} & 0 \\
0 & e^{-s \tau} I_{n-n_{1}}
\end{array}\right)-P \bar{A} Q\right)^{-1}\left(P \Delta A Q+P(\bar{B}+\Delta B) Q e^{-s \tau}\right)\right) \\
& \leq \rho\left(G_{M}\left(\left|P\left(\bar{B}+B_{M}\right) Q\right|\right)\right)<1 .
\end{aligned}
$$

According to (10) and (11), we can get $D(s) \neq 0$ for $\mathfrak{R} e(s) \geq 0$. So when that two conditions in Theorem 3.5 hold, we can obtain that all the roots of the characteristic equation of system (2) have negative real parts, then the interval system (2) is Lyapunov asymptotically stable. Thus the proof is completed.

\section{Numerical Examples}

In this section, some numerical examples are given to demonstrate the effectiveness of those theorems in section 3 .

Example 4.1 Consider the stability of the following linear fractional order neutral system with time delay

$$
\frac{d^{\alpha}}{d t^{\alpha}}(\operatorname{Ex}(t)-C x(t-\tau))=A x(t)-B x(t-\tau)
$$

where $\alpha=\frac{1}{2}$, and

$$
\begin{aligned}
& E=\left(\begin{array}{ll}
2 & 0 \\
0 & 0
\end{array}\right), C=\left(\begin{array}{cc}
1 & 0 \\
0 & -1
\end{array}\right), \\
& A=\left(\begin{array}{cc}
-1 & 0 \\
0 & -1
\end{array}\right), B=\left(\begin{array}{cc}
0.5 & 0 \\
0 & 0.4
\end{array}\right) .
\end{aligned}
$$

Firstly, note that

$F(s, \tau)=s^{\alpha}\left(E-C e^{-s \tau}\right)-A=\left(\begin{array}{cc}s^{\alpha}\left(2-e^{-s \tau}\right)+1 & 0 \\ 0 & s^{\alpha} e^{-s \tau}+1\end{array}\right)$,

So we have $\operatorname{det}(F(s, \tau) \neq 0$ for $\forall \tau>0$ and $\Re e(s) \geq 0$, 


$$
(F(s, \tau))^{-1}=\left(\begin{array}{cc}
\frac{1}{1+s^{\alpha}\left(2-e^{-s \tau}\right)} & 0 \\
0 & \frac{1}{1+s^{\alpha} e^{-s \tau}}
\end{array}\right),
$$

So we can get $F_{M}=\left(\begin{array}{ll}1 & 0 \\ 0 & 1\end{array}\right)$. Since the eigenvalues of matrix $F_{M}|B|$ are $\lambda_{1}=0.5, \lambda_{2}=0.4$, then we can get $\rho\left(F_{M}|B|\right)<1$ easily. Therefore, from Theorem 3.2, we know that the fractional system (12) is Lyapunov stable.

Example 4.2. Consider the stability of the following interval linear fractional order neutral system with time delay

$$
\frac{d^{\alpha}}{d t^{\alpha}}(E x(t)-C x(t-\tau))=A x(t)-B x(t-\tau)
$$

where $\alpha=\frac{1}{2}$, and

$$
\begin{gathered}
E=\left(\begin{array}{ll}
2 & 0 \\
0 & 0
\end{array}\right), C=\left(\begin{array}{cc}
1 & 0 \\
0 & -1
\end{array}\right), A^{1}=\left(\begin{array}{cc}
-1.2 & 0 \\
0 & -1.3
\end{array}\right), \\
A^{2}=\left(\begin{array}{cc}
-0.8 & 0 \\
0 & -0.7
\end{array}\right), B^{1}=\left(\begin{array}{cc}
0.21 & 0 \\
0 & 0.39
\end{array}\right), B^{2}=\left(\begin{array}{cc}
0.72 & 0 \\
0 & 0.5
\end{array}\right) .
\end{gathered}
$$

Firstly, we note that

$$
\begin{gathered}
\bar{A}=\left(\begin{array}{cc}
-1 & 0 \\
0 & -1
\end{array}\right), A_{M}=\left(\begin{array}{cc}
0.2 & 0 \\
0 & 0.3
\end{array}\right), \\
\bar{B}=\left(\begin{array}{cc}
0.465 & 0 \\
0 & 0.445
\end{array}\right), B_{M}=\left(\begin{array}{cc}
0.155 & 0 \\
0 & 0.095
\end{array}\right) .
\end{gathered}
$$

From Example 4.1, we know that

$$
F_{M}=\left(\begin{array}{ll}
1 & 0 \\
0 & 1
\end{array}\right)
$$

So we can get

$$
F_{M}\left(A_{M}+|\bar{B}|+B_{M}\right)=\left(\begin{array}{cc}
0.82 & 0 \\
0 & 0.84
\end{array}\right) .
$$

Since the eigenvalues of matrix $F_{M}\left(A_{M}+|\bar{B}|+B_{M}\right)$ are $\lambda_{1}=0.82, \lambda_{2}=0.84$, then $\rho\left(F_{M}\left(|\bar{B}|+B_{M}\right)<1\right.$. Therefore, from Theorem 3.4, we know that the interval fractional order system (13) is Lyapunov stable.

\section{Conclusions}

In summary, this paper mainly presents some brief sufficient conditions for the stability of a class of linear fractional order neutral system with delay and linear interval fractional order neutral system with delay. The proposed method here is quite different from other ones in literature. Two simple examples also demonstrate that this method is feasible.

\section{References}

[1] I. Podlubuy, Fractional differential equations. New York: Academic Press, 1999.

[2] Ita, J. J. and L. Stixrude, "Petrology, elasticity and composition of the transition zone," Journal of Geophysical Research, vol. 97, pp. 6849-6866, 1992.

[3] A. Oustaloup, X. Morean, M. Nouiuant, "The CRONE Suspension. Control Eng. Pract.," vol. 4 (8), pp.1101-1108. 1996.

[4] O. Side, Electromagnetic theory, Chelsea, New York, 1971.

[5] D. Matignon, "Stability Results on fractional Differential Equations to Control Processing, in: Peocessings of Computational Engineering in Syatems and Application Multiconference," vol. 2, IMACS, IEEE-SMC, pp. 963-968, 1996.

[6] Y. Q. Chen, K. L. Moore, "Analytical Stability Bound for a Class of Delayed Fractional-Order Dynamic Systems," Nonlinear Dynamics, vol. 29, pp. 191-202, 2002.

[7] C. Hwang, C. C. Yi, "Use of Lambert W function to Stability Analysis of Time-Delay System," Portland, OR, USA, pp. 4283-4288. June 8-10, 2005.

[8] K. W. Liu, W. Jiang, "Stability of Fractional Neutral Systems," Advanced in Differential Equations, vol. 78, pp. 1-9, 2014.

[9] J. Chen, D. Xu, B. Shafai, "On Sufficient Conditions for Stability Indenpendent of Delay," IEEE Trans. Automat Control, vol. 40 (9), pp.1675-1680, 1995.

[10] T. Mori, "Criteria for Asymptotic Stability of Linear Time Delay Systems," IEEE Trans. Automat Control, vol. 30 (2), pp. 158-161, 1985.

[11] M. Lazarevic, "Stability and Stabilization of Fractional Order Time Delay Systems," Scientific Technical Review, vol. 61-1, pp. 31-45, 2011.

[12] J. Sabatier, M. Moze, C. Farges, "LMI stability conditions for fractional order systems," Computers and Mathematics with Applications, vol. 59, pp. 1594-1609, 2010.

[13] M. P. Lazarevic, "Finite time stability analysis of fractional control of robotic time-delay systems: Gronwall's approach," Mathematical and Computer Modelling, vol. 49, pp. 475-481, 2009.

[14] Qing-long Han, "Stability of linear neutral system with linear fractional norm-bounded uncertainty," American Control Conference, vol. 4, pp. 2827-2832, 2005.

[15] C. A. Desoer, M. Vidyasagar, Feedback system: input-output properties, Academic press, New York, 1975.

[16] H. Li, S. M. Zhong, H. B. Li, "Stability analysis of Fractional-order systems with time delay," International J. Mathematical, Computational Science and Engineering, vol. 8 (4), pp. 14-17, 2014. 